\title{
Technical note: Equivalent genomic models with a residual polygenic effect
}

\author{
Z. Liu, ${ }^{* 1}$ M. E. Goddard,† B. J. Hayes,† F. Reinhardt, ${ }^{*}$ and R. Reents* \\ *VIT w.V., Heinrich-Schröder-Weg 1, D-27283 Verden, Germany \\ †Dairy Futures Cooperative Research Centre, Bundoora, Melbourne 3083, VIC, Australia
}

\section{ABSTRACT}

Routine genomic evaluations in animal breeding are usually based on either a BLUP with genomic relationship matrix (GBLUP) or single nucleotide polymorphism (SNP) BLUP model. For a multi-step genomic evaluation, these 2 alternative genomic models were proven to give equivalent predictions for genomic reference animals. The model equivalence was verified also for young genotyped animals without phenotypes. Due to incomplete linkage disequilibrium of SNP markers to genes or causal mutations responsible for genetic inheritance of quantitative traits, SNP markers cannot explain all the genetic variance. A residual polygenic effect is normally fitted in the genomic model to account for the incomplete linkage disequilibrium. In this study, we start by showing the proof that the multi-step GBLUP and SNP BLUP models are equivalent for the reference animals, when they have a residual polygenic effect included. Second, the equivalence of both multistep genomic models with a residual polygenic effect was also verified for young genotyped animals without phenotypes. Additionally, we derived formulas to convert genomic estimated breeding values of the GBLUP model to its components, direct genomic values and residual polygenic effect. Third, we made a proof that the equivalence of these 2 genomic models with a residual polygenic effect holds also for single-step genomic evaluation. Both the single-step GBLUP and SNP BLUP models lead to equal prediction for genotyped animals with phenotypes (e.g., reference animals), as well as for (young) genotyped animals without phenotypes. Finally, these 2 single-step genomic models with a residual polygenic effect were proven to be equivalent for estimation of SNP effects, too.

Key words: SNP BLUP model, BLUP with genomic relationship matrix, equivalent model, residual polygenic effect

Received September 14, 2015.

Accepted November 10, 2015.

${ }^{1}$ Corresponding author: Zengting.Liu@vit.de

\section{Technical Note}

Based on the concept of genomic prediction by $\mathrm{Meu}-$ wissen et al. (2001), genomic evaluation and selection have become the method of choice in routine animal breeding practice (Hayes et al., 2009). VanRaden (2008) introduced statistical methods for genomic prediction, including the BLUP with genomic relationship matrix (GBLUP) model and the SNP effect model. Under the assumption of equal variance for all SNP markers, the SNP model has been referred to as a SNP BLUP model. The 2 alternative genomic models have been found in many applications (Meuwissen et al., 2001; VanRaden, 2008; Liu et al., 2011).

Strandén and Garrick (2009) proved that the SNP BLUP and GBLUP models are equivalent for genomic reference animals. Using a different procedure, Taylor (2014) demonstrated that these 2 genomic models are also equivalent for young genotyped animals without own phenotypes. Both papers focused on a genomic model without an explicitly fitted residual polygenic (RPG) effect, which was found to be important to reduce the inflation of genomic prediction with actual data and to account for the incomplete linkage disequilibrium between the SNP markers and genes or causal mutations of analyzed traits (Liu et al., 2011; Su et al., 2014). To our knowledge, no similar proof has been published for the SNP BLUP and GBLUP models with a RPG effect.

The GBLUP model can provide genomic estimated breeding values (GEBV) for reference or young genotyped animals as the sum of direct genomic value (DGV), which represents effects of all SNP genotypes, and RPG effects. However, it is unclear how to obtain the 2 components of GEBV under the GBLUP model. In addition, back solving SNP effect estimates from GEBV of the GBLUP model (Strandén and Garrick, 2009) needs to be updated for the GBLUP model including a RPG effect.

Similar to the development of the multi-step genomic models, 2 groups of single-step genomic model have been developed: single-step GBLUP model (Misztal et al., 2009; Christensen and Lund, 2010; Legarra and Ducrocq, 2012; Su et al., 2012) and single-step SNP 
BLUP model (Legarra and Ducrocq, 2012; Fernando et al., 2014; Liu et al., 2014). To reduce the problem of overestimation of GEBV for young animals, genomic relationship matrix was usually combined with pedigree relationship matrix in the single-step GBLUP model; whereas a RPG effect was fitted in the single-step SNP BLUP model by Liu et al. (2014). As for the multi-step genomic models, we wish to prove that the 2 groups of single-step genomic models with a RPG effect are equivalent.

The objectives of our study were (1) to prove that both the multi-step GBLUP and SNP BLUP models with a RPG effect give equal GEBV for reference animals, (2) to prove that the 2 multi-step genomic models with a RPG effect lead to identical genomic predictions for young animals without phenotypes, and (3) also to prove that the 2 single-step genomic models with a RPG effect result in equivalent prediction for genotyped animals with phenotypes and for genotyped animals without phenotypes.

A mixed linear model is used to analyze phenotypic data of genomic reference animals:

$$
\mathbf{y}=\mathbf{X b}+\mathbf{W}_{2} \mathbf{u}_{2}+\mathbf{e}
$$

where $\mathbf{y}$ is a $n_{\mathrm{T}} \times 1$ vector of corrected phenotypic records, such as deregressed EBV of bulls with daughters (Jairath et al., 1998); $n_{\mathrm{T}}=$ number of phenotype records; $\mathbf{b}$ is a $n_{\mathrm{F}} \times 1$ vector of all fixed effects, $n_{\mathrm{F}}=$ number of all fixed effects; $\mathbf{u}_{2}$ is a $n \times 1$ vector of additive genetic effects, $n=$ number of reference animals; $\mathbf{e}$ is a $n_{\mathrm{T}} \times 1$ vector of residual effects; and $\mathbf{X}$ of order $n_{\mathrm{T}}$ $\times n_{\mathrm{F}}$ and $\mathbf{W}_{2}$ of order $n_{\mathrm{T}} \times n$ are incidence matrices for effects $\mathbf{b}$ and $\mathbf{u}_{2}$, respectively.

The genomic model [1] may be extended to multiple trait evaluations. If a reference animal has phenotype record in at least one trait, it can be included in the reference population even with some traits missing. The multi-trait reference population may be computationally more efficient than a separate genotype subset for each trait, because the model effects can be estimated in parallel.

We assume that $m$ SNP markers selected for genomic evaluation cannot explain all additive genetic variance $\left(\sigma_{u}^{2}\right)$ due to incomplete linkage disequilibrium to causal mutations responsible for the inheritance of the analyzed trait, leaving $k \sigma_{u}^{2}$ residual polygenic variance, where $k$ is the proportion of additive genetic variance not explained by all the $m$ SNP markers. Thus, additive genetic effects of the reference animals can be divided into

$$
\mathbf{u}_{2}=\mathbf{Z g}+\mathbf{a}_{2},
$$

where $\mathbf{g}=m \times 1$ vector of additive genetic effects of the $m$ fitted SNP markers, $\mathbf{a}_{2}=n \times 1$ vector of RPG effects of the reference animals, and $\mathbf{Z}=$ a design matrix of order $n \times m$ containing regression coefficients on genotypes of the reference animals at all $m$ SNP markers: $2-2 p_{j}, 1-2 p_{j},-2 p_{j}$, for genotype $\mathrm{AA}, \mathrm{AB}$, or $\mathrm{BB}$ of the $j$ th marker (VanRaden, 2008), where $p_{j}$ represents allele frequency of the $j$ th SNP marker. Let us denote DGV as

$$
\mathbf{u}_{\mathrm{DGV}}=\mathbf{Z g} \text {. }
$$

Replacing $\mathbf{u}_{2}$ of model [1] with equation [2] gives a SNP BLUP model:

$$
\mathbf{y}=\mathbf{X b}+\mathbf{W}_{2} \mathbf{Z g}+\mathbf{W}_{2} \mathbf{a}_{2}+\mathbf{e} .
$$

Let $\operatorname{var}(\mathbf{e})=\mathbf{R}$. The RPG effects of the reference animals have a normal distribution:

$$
\mathbf{a}_{2} \sim N\left(\mathbf{0}, k \sigma_{u}^{2} \mathbf{A}_{22}\right),
$$

where $\mathbf{A}_{22}$ represents pedigree relationship matrix among the reference animals. Residual polygenic variance parameter $k$ may take any value between 0 and 1 , but excluding 2 boundary values $k=0$ and $k=1$. It is further assumed that additive genetic effects of the SNP markers follow a normal distribution:

$$
\mathbf{g} \sim N\left(\mathbf{0},(1-k) \sigma_{u}^{2} \mathbf{B}\right) .
$$

Under the assumption of uncorrelated SNP effects, B is a diagonal matrix of order of $m \times m$. If all the $m$ SNP markers explain equal additive genetic variance such as in a SNP BLUP model (Meuwissen et al., 2001; VanRaden, 2008; Liu et al., 2011), then

$$
\mathbf{B}=\left(\sum_{j=1}^{m} 2 p_{j}\left(1-p_{j}\right)\right)^{-1} \mathbf{I} .
$$

Assuming zero covariance between $\mathbf{g}$ and $\mathbf{a}_{2}$ :

$$
\operatorname{Cov}\left(\mathbf{g}, \mathbf{a}_{2}\right)=\mathbf{0}
$$

Let us denote observed genomic relationship as

$$
\mathbf{G}_{\mathrm{DGV}}=\mathbf{Z B Z}^{\prime}
$$


As shown in Liu et al. (2014), we have

$$
\begin{aligned}
\operatorname{var}\left(\mathbf{u}_{2}\right) & =\operatorname{var}\left(\mathbf{Z g}+\mathbf{a}_{2}\right)=\left[(1-k) \mathbf{Z B Z} \mathbf{H}^{\prime}+k \mathbf{A}_{22}\right] \sigma_{u}^{2} \\
& =\left[(1-k) \mathbf{G}_{D G V}+k \mathbf{A}_{22}\right] \sigma_{u}^{2}=\sigma_{u}^{2} \mathbf{G}_{22} .
\end{aligned}
$$

From formula [10] we can see that the genomic relationship matrix $\mathbf{G}_{22}$, which was first given in VanRaden (2008), under the SNP BLUP model [4] is a linear function of the observed genomic relationship matrix $\mathbf{G}_{\mathrm{DGV}}$ with a weight $(1-k)$ and the expected pedigree relationship matrix $\mathbf{A}_{22}$ with a weight $k$.

Covariance between the RPG effect $\mathbf{a}_{2}$ and additive genetic effect $\mathbf{u}_{2}$ is

$$
\operatorname{cov}\left(\mathbf{a}_{2}, \mathbf{u}_{2}\right)=\operatorname{cov}\left(\mathbf{a}_{2}, \mathbf{Z g}+\mathbf{a}_{2}\right)=\operatorname{var}\left(\mathbf{a}_{2}\right)=k \sigma_{u}^{2} \mathbf{A}_{22} .
$$

Using the covariance [11], RPG effect $\mathbf{a}_{2}$ can be predicted from GEBV $\hat{\mathbf{u}}_{2}$ with

$$
\begin{aligned}
\hat{\mathbf{a}}_{2} & =\operatorname{cov}\left(\mathbf{a}_{2}, \mathbf{u}_{2}\right)\left[\operatorname{var}\left(\mathbf{u}_{2}\right)\right]^{-1} \hat{\mathbf{u}}_{2} \\
& =k \sigma_{u}^{2} \mathbf{A}_{22} \sigma_{u}^{-2} \mathbf{G}_{22}^{-1} \hat{\mathbf{u}}_{2}=k \mathbf{A}_{22} \mathbf{G}_{22}^{-1} \hat{\mathbf{u}}_{2} .
\end{aligned}
$$

Based on equation [12], we get

$$
\mathbf{A}_{22}^{-1} \hat{\mathbf{a}}_{2}=k \mathbf{G}_{22}^{-1} \hat{\mathbf{u}}_{2} .
$$

In a similar way we can obtain the covariance of $\mathbf{u}_{2}$ with DGV:

$$
\begin{aligned}
\operatorname{cov}\left(\mathbf{Z g}, \mathbf{u}_{2}\right) & =\operatorname{cov}\left(\mathbf{Z g}, \mathbf{Z g}+\mathbf{a}_{2}\right)=\operatorname{var}(\mathbf{Z g}) \\
& =(1-k) \sigma_{u}^{2} \mathbf{Z B Z} \mathbf{Z}^{\prime}=(1-k) \sigma_{u}^{2} \mathbf{G}_{\mathrm{DGV}} .
\end{aligned}
$$

The DGV of the reference animals $\hat{\mathbf{u}}_{\mathrm{DGV}}$ can be predicted from $\hat{\mathbf{u}}_{2}$ with

$$
\begin{aligned}
\hat{\mathbf{u}}_{\mathrm{DGV}} & =\mathbf{Z} \hat{\mathbf{g}}=\operatorname{cov}\left(\mathbf{Z g}, \mathbf{u}_{2}\right)\left[\operatorname{var}\left(\mathbf{u}_{2}\right)\right]^{-1} \hat{\mathbf{u}}_{2} \\
& =(1-k) \sigma_{u}^{2} \mathbf{G}_{\mathrm{DGV}} \sigma_{u}^{-2} \mathbf{G}_{22}^{-1} \hat{\mathbf{u}}_{2}=(1-k) \mathbf{G}_{\mathrm{DGV}} \mathbf{G}_{22}^{-1} \hat{\mathbf{u}}_{2} .
\end{aligned}
$$

Pre-multiplying equation [15] by $\left(\mathbf{G}_{\mathrm{DGV}}\right)^{-1} \mathbf{Z}$ ' B leads to equation for the SNP effects $\mathrm{g}$ :

$$
\hat{\mathbf{g}}=(1-k) \mathbf{B Z} \mathbf{G}_{22}^{-1} \hat{\mathbf{u}}_{2} .
$$

Equation [16], first given by VanRaden (2008), is equivalent to formula [6] in Strandén and Garrick (2009), formula [10] in Taylor (2014), and that in Legarra and Ducrocq (2012). Also equation [16] is identical to the formula [B8] in Liu et al. (2014), considering the difference in the definitions of matrix $\mathbf{B}$. Existence of the inverse matrix $\mathbf{G}_{\mathrm{DGV}}^{-1}=(\mathbf{Z B Z})^{-1}$ is not guaranteed even when fewer reference animals are present than the number of fitted markers in the GBLUP model (i.e., $n<m$ ), if the marker genotypes are not all linearly independent because of linkage disequilibrium or if identical twins are present, and so on. When the number of reference animals exceeds the number of markers, the genomic relationship matrix $\mathbf{G}_{\mathrm{DGV}}=$ ZBZ' will more likely be noninvertible. However, including a residual polygenic effect (see equation [10]) can improve numerical stability. Using equation [16], the following equation can be derived:

$$
\mathbf{B}^{-1} \hat{\mathbf{g}}=(1-k) \mathbf{Z}^{\prime} \mathbf{G}_{22}^{-1} \hat{\mathbf{u}}_{2}
$$

Equations [12] and [15] can be used to convert GEBV to its components, RPG and DGV, respectively, for the reference animals.

To estimate RPG effects of the reference animals using the SNP BLUP model [4], pedigree relationship of the reference animals to their nongenotyped ancestors, denoted with subscript 0 , may be defined as

$$
\left[\begin{array}{ll}
\mathbf{A}_{00} & \mathbf{A}_{02} \\
\mathbf{A}_{20} & \mathbf{A}_{22}
\end{array}\right]
$$

and the inverse of the above pedigree relationship matrix is

$$
\left[\begin{array}{ll}
\mathbf{A}_{00} & \mathbf{A}_{02} \\
\mathbf{A}_{20} & \mathbf{A}_{22}
\end{array}\right]^{-1}=\left[\begin{array}{ll}
\mathbf{A}^{00} & \mathbf{A}^{02} \\
\mathbf{A}^{20} & \mathbf{A}^{22}
\end{array}\right] .
$$

According to the inversion rule of partitioned matrices (Harville, 1997),

$$
\mathbf{A}_{22}^{-1}=\mathbf{A}^{22}-\mathbf{A}^{20}\left(\mathbf{A}^{00}\right)^{-1} \mathbf{A}^{02} .
$$

Henderson's mixed model equations (MME) for the SNP BLUP model [4], by adding equations for RPG of nongenotyped ancestors of the reference animals, are as in equation [20], see the next page, where $\hat{\mathbf{a}}_{0}$ represents RPG of the nongenotyped ancestors of the reference animals. 


$\left[\begin{array}{cccc}\mathbf{X}^{\prime} \mathbf{R}^{-1} \mathbf{X} & \mathbf{X}^{\prime} \mathbf{R}^{-1} \mathbf{W}_{2} & \mathbf{0} & \mathbf{X}^{\prime} \mathbf{R}^{-1} \mathbf{W}_{2} \mathbf{Z} \\ & \mathbf{W}_{2}{ }^{\prime} \mathbf{R}^{-1} \mathbf{W}_{2}+\frac{1}{k} \sigma_{u}^{-2} \mathbf{A}^{22} & \frac{1}{k} \sigma_{u}^{-2} \mathbf{A}^{20} & \mathbf{W}_{2} \mathbf{R}^{-1} \mathbf{W}_{2} \mathbf{Z} \\ & & \frac{1}{k} \sigma_{u}^{-2} \mathbf{A}^{00} & \mathbf{0} \\ \text { symm. } & & & \mathbf{Z}^{\prime} \mathbf{W}_{2}{ }^{\prime} \mathbf{R}^{-1} \mathbf{W}_{2} \mathbf{Z}+\frac{1}{1-k} \sigma_{u}^{-2} \mathbf{B}^{-1}\end{array}\right]\left[\begin{array}{c}\hat{\mathbf{b}} \\ \hat{\mathbf{a}}_{2} \\ \hat{\mathbf{a}}_{0} \\ \hat{\mathbf{g}}\end{array}\right]=\left[\begin{array}{c}\mathbf{X}^{\prime} \mathbf{R}^{-1} \mathbf{y} \\ \mathbf{W}_{2}{ }^{\prime} \mathbf{R}^{-1} \mathbf{y} \\ \mathbf{0} \\ \mathbf{Z}^{\prime} \mathbf{W}_{2}{ }^{\prime} \mathbf{R}^{-1} \mathbf{y}\end{array}\right]$,

$$
\left[\begin{array}{ccc}
\mathbf{X}^{\prime} \mathbf{R}^{-1} \mathbf{X} & \mathbf{X}^{\prime} \mathbf{R}^{-1} \mathbf{W}_{2} & \mathbf{X}^{\prime} \mathbf{R}^{-1} \mathbf{W}_{2} \mathbf{Z} \\
& \mathbf{W}_{2}{ }^{\prime} \mathbf{R}^{-1} \mathbf{W}_{2}+\frac{1}{k} \sigma_{u}^{-2} \mathbf{A}_{22}^{-1} & \mathbf{W}_{2}{ }^{\prime} \mathbf{R}^{-1} \mathbf{W}_{2} \mathbf{Z} \\
\text { symm. } & & \mathbf{Z}^{\prime} \mathbf{W}_{2} \mathbf{R}^{-1} \mathbf{W}_{2} \mathbf{Z}+\frac{1}{1-k} \sigma_{u}^{-2} \mathbf{B}^{-1}
\end{array}\right]\left[\begin{array}{c}
\hat{\mathbf{b}} \\
\hat{\mathbf{a}}_{2} \\
\hat{\mathbf{g}}
\end{array}\right]=\left[\begin{array}{c}
\mathbf{X}^{\prime} \mathbf{R}^{-1} \mathbf{y} \\
\mathbf{W}_{2}{ }^{\prime} \mathbf{R}^{-1} \mathbf{y} \\
\mathbf{Z}^{\prime} \mathbf{W}_{2}{ }^{\prime} \mathbf{R}^{-1} \mathbf{y}
\end{array}\right]
$$

After absorbing $\hat{\mathbf{a}}_{0}$ in $\hat{\mathbf{a}}_{2}$ and applying equation [19], MME [20] are simplified as in equation [21], see above.

By restricting to only the genotyped reference animals, Henderson's MME of the GBLUP model (VanRaden, 2008) are

$$
\left[\begin{array}{cc}
\mathbf{X}^{\prime} \mathbf{R}^{-1} \mathbf{X} & \mathbf{X}^{\prime} \mathbf{R}^{-1} \mathbf{W}_{2} \\
\text { symm. } & \mathbf{W}_{2} \mathbf{R}^{-1} \mathbf{W}_{2}+\sigma_{u}^{-2} \mathbf{G}_{22}^{-1}
\end{array}\right]\left[\begin{array}{c}
\hat{\mathbf{b}} \\
\hat{\mathbf{u}}_{2}
\end{array}\right]=\left[\begin{array}{c}
\mathbf{X}^{\prime} \mathbf{R}^{-1} \mathbf{y} \\
\mathbf{W}_{2}{ }^{\prime} \mathbf{R}^{-1} \mathbf{y}
\end{array}\right] .
$$

If we wish to jointly evaluate a group of young genotyped animals that have no own phenotypes, denoted with subscript $y$, the following genomic relationship matrices and their inverse are required between the reference and young animals:

$$
\left[\begin{array}{ll}
\mathbf{G}_{22} & \mathbf{G}_{2 y} \\
\mathbf{G}_{y 2} & \mathbf{G}_{y y}
\end{array}\right]^{-1}=\left[\begin{array}{ll}
\mathbf{G}^{22} & \mathbf{G}^{2 y} \\
\mathbf{G}^{y 2} & \mathbf{G}^{y y}
\end{array}\right]
$$

By adding GEBV of the young animals, $\mathbf{u}_{y}$, MME [22] are extended to

$$
\left[\begin{array}{ccc}
\mathbf{X}^{\prime} \mathbf{R}^{-1} \mathbf{X} & \mathbf{X}^{\prime} \mathbf{R}^{-1} \mathbf{W}_{2} & \mathbf{0} \\
\mathbf{W}_{2} \mathbf{R}^{-1} \mathbf{X} & \mathbf{W}_{2}^{\prime} \mathbf{R}^{-1} \mathbf{W}_{2}+\sigma_{u}^{-2} \mathbf{G}^{22} & \sigma_{u}^{-2} \mathbf{G}^{2 y} \\
\mathbf{0} & \sigma_{u}^{-2} \mathbf{G}^{y 2} & \sigma_{u}^{-2} \mathbf{G}^{y y}
\end{array}\right]\left[\begin{array}{c}
\hat{\mathbf{b}} \\
\hat{\mathbf{u}}_{2} \\
\hat{\mathbf{u}}_{y}
\end{array}\right]=\left[\begin{array}{c}
\mathbf{X}^{\prime} \mathbf{R}^{-1} \mathbf{y} \\
\mathbf{W}_{2} \mathbf{R}^{-1} \mathbf{y} \\
\mathbf{0}
\end{array}\right] .
$$

From equation [24] for the young genotyped animals, we can see that GEBV of the young animals, $\hat{\mathbf{u}}_{y}$, can be predicted from the GEBV of the reference animals, $\hat{\mathbf{u}}_{2}$, and their genomic relationship to the reference animals:

$$
\hat{\mathbf{u}}_{y}=-\left(\mathbf{G}^{y y}\right)^{-1} \mathbf{G}^{y 2} \hat{\mathbf{u}}_{2}
$$

Applying equation [25], equation [24] for $\hat{\mathbf{u}}_{2}$ becomes

$$
\begin{aligned}
& \mathbf{W}_{2}{ }^{\prime} \mathbf{R}^{-1} \mathbf{X} \hat{\mathbf{b}}+\mathbf{W}_{2}{ }^{\prime} \mathbf{R}^{-1} \mathbf{W}_{2} \hat{\mathbf{u}}_{2} \\
& +\sigma_{u}^{-2}\left[\mathbf{G}^{22} \hat{\mathbf{u}}_{2}-\mathbf{G}^{2 y}\left(\mathbf{G}^{y y}\right)^{-1} \mathbf{G}^{y 2} \hat{\mathbf{u}}_{2}\right]=\mathbf{W}_{2}{ }^{\prime} \mathbf{R}^{-1} \mathbf{y} .
\end{aligned}
$$

Because $\mathbf{G}^{22}-\mathbf{G}^{2 y}\left(\mathbf{G}^{y y}\right)^{-1} \mathbf{G}^{y 2}=\mathbf{G}_{22}^{-1}$ from the inverse of partitioned matrices [23], we can see that the GEBV of the reference animals, $\hat{\mathbf{u}}_{2}$, from the 2 sets of mixed model equations [22] and [24] are equal, regardless of whether or not the young genotyped animals without phenotypes are jointly evaluated, which has long been accepted as a fact. The GEBV of the reference animals $\hat{\mathbf{u}}_{2}$ being equal from both MME are only true, as nongenotyped relatives, with phenotypes, of the young animals are ignored in the multi-step genomic models [1] and [4]. In contrast, single-step genomic models (Misztal et al., 2009; Christensen and Lund, 2010; Legarra and Ducrocq, 2012; Liu et al., 2014) properly consider the relationship between the young genotyped animals and their nongenotyped relatives with phenotype information.

The DGV of the reference animals can be estimated from their GEBV of the GBLUP model using equation [15]: $\hat{\mathbf{u}}_{\mathrm{DGV}}=(1-k) \mathbf{G}_{\mathrm{DGV}} \mathbf{G}_{22}^{-1} \hat{\mathbf{u}}_{2}$. Please note that the GEBV are obtained directly by solving MME [22] or [24], and they are not necessarily equal to officially published genomic evaluations as in some countries that also contain the conventional parental average.

Furthermore, additive genetic effects of the SNP markers can be back solved using equation [16]. Likewise, RPG of the reference animals can be estimated from their GEBV of the GBLUP model using equation [12]: $\hat{\mathbf{a}}=k \mathbf{A}_{22} \mathbf{G}_{22}^{-1} \hat{\mathbf{u}}_{2}$.

Strandén and Garrick (2009) proved that GBLUP and SNP BLUP genomic models, without a residual polygenic effect explicitly fitted, are equivalent for predicting GEBV of reference animals. We wish to prove the equivalence also in case of the genomic models including a residual polygenic effect. Mixed model equa- 
tions [21] of the SNP BLUP model and [22] of the GBLUP model must result in equal genomic predictions for the reference animals, if the 2 genomic models are equivalent. If we can show that the 2 sets of MME give equivalent estimates of all the random effects, then estimates of the same fixed effects must be equal as well. First, we demonstrate that the equation for SNP effects of MME [21] leads to estimates of GEBV of the reference animals as the equation $\hat{\mathbf{u}}_{2}$ of GBLUP MME [22]. The equation for $\hat{\mathbf{g}}$ from the SNP BLUP model MME [21] is

$$
\begin{aligned}
& \mathbf{Z}^{\prime} \mathbf{W}_{2}{ }^{\prime} \mathbf{R}^{-1} \mathbf{X} \hat{\mathbf{b}}+\mathbf{Z}^{\prime} \mathbf{W}_{2}{ }^{\prime} \mathbf{R}^{-1} \mathbf{W}_{2} \hat{\mathbf{a}}_{2}+\mathbf{Z}^{\prime} \mathbf{W}_{2}{ }^{\prime} \mathbf{R}^{-1} \mathbf{W}_{2} \mathbf{Z} \hat{\mathbf{g}} \\
& +\frac{1}{1-k} \sigma_{u}^{-2} \mathbf{B}^{-1} \hat{\mathbf{g}}=\mathbf{Z}^{\prime} \mathbf{W}_{2}{ }^{\prime} \mathbf{R}^{-1} \mathbf{y} .
\end{aligned}
$$

Using formula [2] and equation [17], equation [27] becomes

$$
\begin{array}{r}
\mathbf{Z}^{\prime} \mathbf{W}_{2}{ }^{\prime} \mathbf{R}^{-1} \mathbf{X} \hat{\mathbf{b}}+\mathbf{Z}^{\prime} \mathbf{W}_{2}{ }^{\prime} \mathbf{R}^{-1} \mathbf{W}_{2} \hat{\mathbf{u}}_{2} \\
+\sigma_{u}^{-2} \mathbf{Z}^{\prime} \mathbf{G}_{22}^{-1} \hat{\mathbf{u}}_{2}=\mathbf{Z}^{\prime} \mathbf{W}_{2}{ }^{\prime} \mathbf{R}^{-1} \mathbf{y}
\end{array}
$$

Pre-multiplying both sides of equation [28] by $\left(\mathbf{Z B Z}^{\prime}\right)^{-1} \mathbf{Z}^{\prime} \mathbf{B}=\mathbf{G}_{D G V}^{-1} \mathbf{Z}^{\prime} \mathbf{B}$ results in

$$
\mathbf{W}_{2}{ }^{\prime} \mathbf{R}^{-1} \mathbf{X} \hat{\mathbf{b}}+\mathbf{W}_{2}{ }^{\prime} \mathbf{R}^{-1} \mathbf{W}_{2} \hat{\mathbf{u}}_{2}+\sigma_{u}^{-2} \mathbf{G}_{22}^{-1} \hat{\mathbf{u}}_{2}=\mathbf{W}_{2}{ }^{\prime} \mathbf{R}^{-1} \mathbf{y}
$$

which is identical to GEBV equation in GBLUP MME [22]. With regard to the existence of the inverse matrix $\left(\mathbf{Z B Z}^{\prime}\right)^{-1}=\mathbf{G}_{\mathrm{DGV}}^{-1}$, we already have given explanation above. Now we have proven that the SNP effect equation of the SNP BLUP model MME leads to GEBV of the reference animals as the GBLUP model MME [22].

Second, we wish to prove that equation of RPG effects in MME [21],

$$
\begin{gathered}
\mathbf{W}_{2}{ }^{\prime} \mathbf{R}^{-1} \mathbf{X} \hat{\mathbf{b}}+\mathbf{W}_{2}{ }^{\prime} \mathbf{R}^{-1} \mathbf{W}_{2} \hat{\mathbf{a}}_{2}+\frac{1}{k} \sigma_{u}^{-2} \mathbf{A}_{22}^{-1} \hat{\mathbf{a}}_{2} \\
+\mathbf{W}_{2}{ }^{\prime} \mathbf{R}^{-1} \mathbf{W}_{2} \mathbf{Z} \hat{\mathbf{g}}=\mathbf{W}_{2}{ }^{\prime} \mathbf{R}^{-1} \mathbf{y}
\end{gathered}
$$

must yield GEBV for the reference animals as the GBLUP model. Using formula [2], $\mathbf{u}_{2}=\mathbf{Z g}+\mathbf{a}_{2}$, and equation [13], $\mathbf{A}_{22}^{-1} \hat{\mathbf{a}}_{2}=k \mathbf{G}_{22}^{-1} \hat{\mathbf{u}}_{2}$, the above equation [30] changes to

$$
\mathbf{W}_{2}{ }^{\prime} \mathbf{R}^{-1} \mathbf{X} \hat{\mathbf{b}}+\mathbf{W}_{2}{ }^{\prime} \mathbf{R}^{-1} \mathbf{W}_{2} \hat{\mathbf{u}}_{2}+\sigma_{u}^{-2} \mathbf{G}_{22}^{-1} \hat{\mathbf{u}}_{2}=\mathbf{W}_{2}{ }^{\prime} \mathbf{R}^{-1} \mathbf{y}
$$

which is identical to equation $\hat{\mathbf{u}}_{2}$ for GEBV of the reference animals under the GBLUP model.

Taylor (2014) successfully proved the equivalence of the 2 genomic models, though no residual polygenic effect was explicitly fitted, for young genotyped animals having no own phenotypes. We wish to demonstrate that this equivalence holds also for the genomic models with a residual polygenic effect included. As for the reference animals, we would like to know if the 2 components of GEBV, DGV, and RPG are equal for young genotyped animals without phenotypes between the SNP BLUP and GBLUP models. Similar to formula [23] denoting genomic relationship between the young and reference animals, we have also for pedigree relationship between the 2 groups of animals:

$$
\left[\begin{array}{ll}
\mathbf{A}_{22} & \mathbf{A}_{2 y} \\
\mathbf{A}_{y 2} & \mathbf{A}_{y y}
\end{array}\right]^{-1}=\left[\begin{array}{ll}
\mathbf{A}^{22} & \mathbf{A}^{2 y} \\
\mathbf{A}^{y 2} & \mathbf{A}^{y y}
\end{array}\right] .
$$

In analogy to the genomic relationship matrix among the reference animals (equations [9] and [10]), let us define the genomic relationship matrix of the young with the reference animals as

$$
\mathbf{G}_{y 2}=(1-k) \mathbf{Z}_{y} \mathbf{B Z}^{\prime}+k \mathbf{A}_{y 2}=(1-k) \mathbf{G}_{y 2_{-} D G V}+k \mathbf{A}_{y 2} .
$$

From formula [33] we can see that the genomic relationship matrix between the young and reference animals is a weighted average of the observed genomic relationship matrix $\mathbf{G}_{y 2 \_ \text {DGV }}$ and expected pedigree relationship matrix $\mathbf{A}_{y 2}$. Applying the inversion rule of partitioned matrix (Harville, 1997) to equation [23], we get

$$
\mathbf{G}_{y 2} \mathbf{G}_{22}^{-1}+\left(\mathbf{G}^{y y}\right)^{-1} \mathbf{G}^{y 2}=\mathbf{0} .
$$

Using equation [34], GEBV of the young animals are predicted from GEBV of the reference animals (equation [25]):

$$
\begin{gathered}
\hat{\mathbf{u}}_{y}=-\left(\mathbf{G}^{y y}\right)^{-1} \mathbf{G}^{y 2} \hat{\mathbf{u}}_{2}=\mathbf{G}_{y 2} \mathbf{G}_{22}^{-1} \hat{\mathbf{u}}_{2}, \\
\hat{\mathbf{u}}_{y}=\left[(1-k) \mathbf{G}_{y 2 \_} D G V+k \mathbf{A}_{y 2}\right] \mathbf{G}_{22}^{-1} \hat{\mathbf{u}}_{2} \\
=(1-k) \mathbf{G}_{y 2 \_} D G V \mathbf{G}_{22}^{-1} \hat{\mathbf{u}}_{2}+k \mathbf{A}_{y 2} \mathbf{G}_{22}^{-1} \hat{\mathbf{u}}_{2} .
\end{gathered}
$$

From the above equation [35], $\hat{\mathbf{u}}_{y}=\mathbf{G}_{y 2} \mathbf{G}_{22}^{-1} \hat{\mathbf{u}}_{2}$, we can see that GEBV of a young animal is not influenced by 
other simultaneously evaluated young animals because its GEBV depends only on GEBV of all the reference animals and its genomic relationships to the reference animals. The equation [35], $\hat{\mathbf{u}}_{y}=\mathbf{G}_{y 2} \mathbf{G}_{22}^{-1} \hat{\mathbf{u}}_{2}$, also tells us that GEBV of the young animals can be calculated in a posterior step after solving the MME [22] with only the reference animals included. This posterior calculation of GEBV for young animals may have a numerical advantage than solving the complete MME [24], when the number of young genotyped animals is significantly higher than the number of reference animals. Equation [36] shows that GEBV of the young animals are a linear function of their DGV and RPG effects.

Having solved equation [21] for all the effects of the SNP BLUP model [4], DGV of the young genotyped animals can be simply calculated as $\mathbf{Z}_{y} \hat{\mathbf{g}}$. Replacing $\hat{\mathbf{g}}$ in $\mathbf{Z}_{y} \hat{\mathbf{g}}$ by equation [16] leads to

$$
\mathbf{Z}_{y} \hat{\mathbf{g}}=(1-k) \mathbf{Z}_{y} \mathbf{B Z} \mathbf{Z}^{\prime} \mathbf{G}_{22}^{-1} \hat{\mathbf{u}}_{2}=(1-k) \mathbf{G}_{y 2 \_\mathrm{DGV}} \mathbf{G}_{22}^{-1} \hat{\mathbf{u}}_{2} .
$$

Therefore, DGV of the young animals estimated using equation [37] of the SNP BLUP model are equal to the first DGV component of GEBV of the GBLUP model in equation [36].

Based on the RPG effect estimates of the reference animals and the pedigree relationship of the young to the reference animals, we can predict RPG of the young animals for the SNP BLUP model [4] as

$$
\begin{aligned}
\hat{\mathbf{a}}_{y} & =\operatorname{cov}\left(\mathbf{a}_{y}, \mathbf{a}_{2}\right)\left[\operatorname{var}\left(\mathbf{a}_{2}\right)\right]^{-1} \hat{\mathbf{a}}_{2} \\
& =k \sigma_{u}^{2} \mathbf{A}_{y 2} \frac{1}{k} \sigma_{u}^{-2} \mathbf{A}_{22}^{-1} \hat{\mathbf{a}}_{2}=\mathbf{A}_{y 2} \mathbf{A}_{22}^{-1} \hat{\mathbf{a}}_{2} .
\end{aligned}
$$

Using equation [13] we obtain RPG of the young animals as

$$
\hat{\mathbf{a}}_{y}=\mathbf{A}_{y 2} \mathbf{A}_{22}^{-1} \hat{\mathbf{a}}_{2}=k \mathbf{A}_{y 2} \mathbf{G}_{22}^{-1} \hat{\mathbf{u}}_{2} .
$$

Equation [39] shows the relation between the RPG effects of the young animals and RPG and GEBV of the reference animals. However, equation [39] is a computationally very inefficient method to compute RPG for the young animals for large genotyped populations. An efficient way to calculate the RPG effects of the young animals is to include the young genotyped animals in the SNP effect estimation with MME [21] using Henderson's sparse inverse of relationship matrix.

Comparing equation [39] to [36], we can draw a conclusion that RPG effects of the young animals estimated with equation [39] of the SNP BLUP model are equal to the second, RPG component of GEBV of the young animals in equation [36] of the GBLUP model. Summing the DGV from equation [37] and RPG effects from equation [39], we can see that GEBV of the young genotyped animals under the SNP BLUP model are equal to those under the GBLUP model (equation [36]).

In contrast to the genomic model [1] or [4], a singlestep genomic model analyses genotypes and phenotypes of all animals, genotyped or not, together with all pedigree information (Misztal et al., 2009; Christensen and Lund, 2010):

$$
\mathbf{y}=\mathbf{X b}+\mathbf{Z}_{\mathrm{p}} \mathbf{p}+\mathbf{W u}+\mathbf{e}
$$

where $\mathbf{y}$ is an $n_{\mathrm{T}} \times 1$ vector of phenotypic data; $\mathbf{p}$ is an $n_{\mathrm{p}} \times 1$ vector of nongenetic random effects (e.g., permanent environmental effects of cows); $\mathbf{u}$ is an $n \times$ 1 vector of additive genetic effects, $n=$ total number of animals (genotyped plus nongenotyped); $\mathbf{Z}_{\mathrm{p}}$ of order $n_{\mathrm{T}}$ $\times n_{\mathrm{p}}$ and $\mathbf{W}$ of order $n_{\mathrm{T}} \times n$ are incidence matrices of effects $\mathbf{p}$ and $\mathbf{u}$, respectively. We assume that

$$
\operatorname{var}(\mathbf{p})=\mathbf{I} \sigma_{p}^{2},
$$

where $\sigma_{p}^{2}$ represents variance of the nongenetic random effects.

It is assumed that 2 groups of animals are to be evaluated: group 1 (without genotype data) and group 2 (with genotype data available). In contrast to the multi-step genomic models [1] or [4], the group 2 includes, besides reference animals, genotyped animals without phenotypes. The relationship between $n_{\mathrm{g}}$ genotyped animals in group 2 and $n-n_{\mathrm{g}}$ nongenotyped animals in group 1 is defined as

$$
\mathbf{A}=\left[\begin{array}{ll}
\mathbf{A}_{11} & \mathbf{A}_{12} \\
\mathbf{A}_{21} & \mathbf{A}_{22}
\end{array}\right]
$$

and its inverse is

$$
\left[\begin{array}{ll}
\mathbf{A}_{11} & \mathbf{A}_{12} \\
\mathbf{A}_{21} & \mathbf{A}_{22}
\end{array}\right]^{-1}=\left[\begin{array}{ll}
\mathbf{A}^{11} & \mathbf{A}^{12} \\
\mathbf{A}^{21} & \mathbf{A}^{22}
\end{array}\right] .
$$

Following equation [2], $\mathbf{u}_{2}=\mathbf{Z g}+\mathbf{a}_{2}$, the single-step genomic model [40] becomes (Liu et al., 2014):

$$
\mathbf{y}=\mathbf{X b}+\mathbf{Z}_{p} \mathbf{p}+\mathbf{W Z g}+\mathbf{W a}+\mathbf{e} .
$$

Same as Liu et al. (2014), we define a vector $\mathbf{h}$ of order $n+m$ as 


$$
\left[\begin{array}{cccc}
\mathbf{X}^{\prime} \mathbf{R}^{-1} \mathbf{X} & \mathbf{X}^{\prime} \mathbf{R}^{-1} \mathbf{Z}_{p} & \mathbf{X}^{\prime} \mathbf{R}^{-1} \mathbf{W}_{1} & \mathbf{X}^{\prime} \mathbf{R}^{-1} \mathbf{W}_{2} \\
& \mathbf{Z}_{p}^{\prime} \mathbf{R}^{-1} \mathbf{Z}_{p}+\sigma_{p}^{-2} \mathbf{I} & \mathbf{Z}_{p}{ }^{\prime} \mathbf{R}^{-1} \mathbf{W}_{1} & \mathbf{Z}_{p}{ }^{\prime} \mathbf{R}^{-1} \mathbf{W}_{2} \\
& & \mathbf{W}_{1}^{\prime} \mathbf{R}^{-1} \mathbf{W}_{1}+\sigma_{u}^{-2} \mathbf{A}^{11} & \sigma_{u}^{-2} \mathbf{A}^{12} \\
\text { symm. } & & & \mathbf{W}_{2}{ }^{\prime} \mathbf{R}^{-1} \mathbf{W}_{2}+\sigma_{u}^{-2}\left(\mathbf{A}^{22}+\mathbf{G}_{22}^{-1}-\mathbf{A}_{22}^{-1}\right)
\end{array}\right]\left[\begin{array}{c}
\hat{\mathbf{b}} \\
\hat{\mathbf{p}} \\
\hat{\mathbf{u}}_{1} \\
\hat{\mathbf{u}}_{2}
\end{array}\right]=\left[\begin{array}{c}
\mathbf{X}^{\prime} \mathbf{R}^{-1} \mathbf{y} \\
\mathbf{Z}_{p}{ }^{\prime} \mathbf{R}^{-1} \mathbf{y} \\
\mathbf{W}_{1}{ }^{\prime} \mathbf{R}^{-1} \mathbf{y} \\
\mathbf{W}_{2} \mathbf{R}^{-1} \mathbf{y}
\end{array}\right]
$$

$$
\begin{aligned}
& {\left[\begin{array}{ccccc}
\mathbf{X}^{\prime} \mathbf{R}^{-1} \mathbf{X} & \mathbf{X}^{\prime} \mathbf{R}^{-1} \mathbf{Z}_{p} & \mathbf{X}^{\prime} \mathbf{R}^{-1} \mathbf{W}_{1} & \mathbf{X}^{\prime} \mathbf{R}^{-1} \mathbf{W}_{2} & \mathbf{0} \\
& \mathbf{Z}_{p}{ }^{\prime} \mathbf{R}^{-1} \mathbf{Z}_{p}+\sigma_{p}^{-2} \mathbf{I} & \mathbf{Z}_{p}{ }^{\prime} \mathbf{R}^{-1} \mathbf{W}_{1} & \mathbf{Z}_{p}{ }^{\prime} \mathbf{R}^{-1} \mathbf{W}_{2} & \mathbf{0} \\
& & \mathbf{W}_{1}{ }^{\prime} \mathbf{R}^{-1} \mathbf{W}_{1}+\sigma_{u}^{-2} \mathbf{A}^{11} & \sigma_{u}^{-2} \mathbf{A}^{12} & \mathbf{0} \\
& & & \mathbf{W}_{2}{ }^{\prime} \mathbf{R}^{-1} \mathbf{W}_{2}+\sigma_{u}^{-2}\left(\mathbf{A}^{22}+\left(\frac{1}{k}-1\right) \mathbf{A}_{22}^{-1}\right) & -\sigma_{u}^{-2} \frac{1}{k} \mathbf{A}_{22}^{-1} \mathbf{Z} \\
\text { symm. } & & & \sigma_{u}^{-2}\left(\frac{1}{1-k} \mathbf{B}^{-1}+\frac{1}{k} \mathbf{Z}^{\prime} \mathbf{A}_{22}^{-1} \mathbf{Z}\right)
\end{array}\right]} \\
& *\left[\begin{array}{c}
\hat{\mathbf{b}} \\
\hat{\mathbf{p}} \\
\hat{\mathbf{u}}_{1} \\
\hat{\mathbf{u}}_{2} \\
\hat{\mathbf{g}}
\end{array}\right]=\left[\begin{array}{c}
\mathbf{X}^{\prime} \mathbf{R}^{-1} \mathbf{y} \\
\mathbf{Z}_{p}{ }^{\prime} \mathbf{R}^{-1} \mathbf{y} \\
\mathbf{W}_{1}{ }^{\prime} \mathbf{R}^{-1} \mathbf{y} \\
\mathbf{W}_{2}{ }^{\prime} \mathbf{R}^{-1} \mathbf{y} \\
\mathbf{0}
\end{array}\right] .
\end{aligned}
$$

$$
\operatorname{var}(\mathbf{h})=\operatorname{var}\left[\begin{array}{c}
\mathbf{u}_{1} \\
\mathbf{u}_{2} \\
\mathbf{g}
\end{array}\right]=\mathbf{H} \sigma_{u}^{2},
$$

where the inverse of $\mathbf{H}$ matrix is (Liu et al., 2014):

$$
\mathbf{H}^{-1}=\left[\begin{array}{ccc}
\mathbf{A}^{11} & \mathbf{A}^{12} & \mathbf{0} \\
\mathbf{A}^{21} & \mathbf{A}^{22}+\left(\frac{1}{k}-1\right) \mathbf{A}_{22}^{-1} & -\frac{1}{k} \mathbf{A}_{22}^{-1} \mathbf{Z} \\
\mathbf{0} & -\frac{1}{k} \mathbf{Z}^{\prime} \mathbf{A}_{22}^{-1} & \frac{1}{1-k} \mathbf{B}^{-1}+\frac{1}{k} \mathbf{Z}^{\prime} \mathbf{A}_{22}^{-1} \mathbf{Z}
\end{array}\right] .
$$

Note that the $\mathbf{H}$ inverse matrix [46] differs with the inverse matrix in Liu et al. (2014) only in the diagonal block for SNP effects due to the different definitions of matrix $\mathbf{B}$. However, the $\mathbf{H}$ inverse matrix in [46] is equivalent to the one in Liu et al. (2014).

For the single-step GBLUP model [40], Henderson's MME (Misztal et al., 2009; Christensen and Lund, 2010) are as in equation [47], see above, where $\mathbf{W}_{1}$ and $\mathbf{W}_{2}$ represent the incidence matrix for additive genetic effects of nongenotyped $\left(\mathbf{u}_{1}\right)$ and genotyped animals $\left(\mathbf{u}_{2}\right)$, respectively.

Same as given in Liu et al. (2014), MME of the singlestep SNP BLUP model [44] are as in [48], see above. Note that MME of the single-step SNP BLUP model
[48] differs only in diagonal block for the SNP marker effects due to the difference in definitions of matrix $\mathbf{B}$ between this paper and Liu et al. (2014). However, both sets of MME give identical solutions to GEBV.

Although Liu et al. (2014) compared the single-step GBLUP model (Legarra and Ducrocq, 2012) and the single-step SNP BLUP model, the comparison was limited, without differentiating different groups of genotyped animals. As demonstrated above for the multi-step genomic models, we would like to prove as well that the 2 single-step models are equivalent for genotyped animals with phenotypes, including genomic reference animals. Comparing MME [47] to [48], we can easily see that all the equations will be equal between the 2 sets of $\mathrm{MME}$, if the $\mathbf{u}_{2}$ equations for genotyped animals with phenotypes are identical.

From MME [47], the $\mathbf{u}_{2}$ equations for the genotyped animals with phenotypes (e.g., genotyped cows with own records) under the single-step GBLUP model are

$$
\begin{aligned}
& \mathbf{W}_{2}{ }^{\prime} \mathbf{R}^{-1} \mathbf{X} \hat{\mathbf{b}}+\mathbf{W}_{2}{ }^{\prime} \mathbf{R}^{-1} \mathbf{Z}_{p} \hat{\mathbf{p}}+\sigma_{u}^{-2} \mathbf{A}^{21} \hat{\mathbf{u}}_{1}+\mathbf{W}_{2}{ }^{\prime} \mathbf{R}^{-1} \mathbf{W}_{2} \hat{\mathbf{u}}_{2} \\
& +\sigma_{u}^{-2}\left(\mathbf{A}^{22}+\mathbf{G}_{22}^{-1}-\mathbf{A}_{22}^{-1}\right) \hat{\mathbf{u}}_{2}=\mathbf{W}_{2}{ }^{\prime} \mathbf{R}^{-1} \mathbf{y} .
\end{aligned}
$$

Corresponding equations for the genotyped animals with phenotypes under the single-step SNP BLUP model are taken from MME [48] as 


$$
\begin{aligned}
& \mathbf{W}_{2}{ }^{\prime} \mathbf{R}^{-1} \mathbf{X} \hat{\mathbf{b}}+\mathbf{W}_{2}{ }^{\prime} \mathbf{R}^{-1} \mathbf{Z}_{p} \hat{\mathbf{p}}+\sigma_{u}^{-2} \mathbf{A}^{21} \hat{\mathbf{u}}_{1}+\mathbf{W}_{2}{ }^{\prime} \mathbf{R}^{-1} \mathbf{W}_{2} \hat{\mathbf{u}}_{2} \\
& +\sigma_{u}^{-2}\left(\mathbf{A}^{22}+\left(\frac{1}{k}-1\right) \mathbf{A}_{22}^{-1}\right) \hat{\mathbf{u}}_{2}-\frac{1}{k} \sigma_{u}^{-2} \mathbf{A}_{22}^{-1} \mathbf{Z} \hat{\mathbf{g}}=\mathbf{W}_{2}{ }^{\prime} \mathbf{R}^{-1} \mathbf{y}
\end{aligned}
$$

and because $\mathbf{a}_{2}=\mathbf{u}_{2}-\mathbf{Z g}$, equation [50] becomes

$$
\begin{gathered}
\mathbf{W}_{2}{ }^{\prime} \mathbf{R}^{-1} \mathbf{X} \hat{\mathbf{b}}+\mathbf{W}_{2}{ }^{\prime} \mathbf{R}^{-1} \mathbf{Z}_{p} \hat{\mathbf{p}}+\sigma_{u}^{-2} \mathbf{A}^{21} \hat{\mathbf{u}}_{1}+\mathbf{W}_{2}{ }^{\prime} \mathbf{R}^{-1} \mathbf{W}_{2} \hat{\mathbf{u}}_{2} \\
+\sigma_{u}^{-2}\left(\mathbf{A}^{22}-\mathbf{A}_{22}^{-1}\right) \hat{\mathbf{u}}_{2}+\frac{1}{k} \sigma_{u}^{-2} \mathbf{A}_{22}^{-1} \hat{\mathbf{a}}_{2}=\mathbf{W}_{2}{ }^{\prime} \mathbf{R}^{-1} \mathbf{y}
\end{gathered}
$$

Applying equation [13], $\mathbf{A}_{22}^{-1} \hat{\mathbf{a}}_{2}=k \mathbf{G}_{22}^{-1} \hat{\mathbf{u}}_{2}$, equation $\mathbf{u}_{2}$ of the single-step SNP BLUP model is finally simplified as

$$
\begin{gathered}
\mathbf{W}_{2}{ }^{\prime} \mathbf{R}^{-1} \mathbf{X} \hat{\mathbf{b}}+\mathbf{W}_{2}{ }^{\prime} \mathbf{R}^{-1} \mathbf{Z}_{p} \hat{\mathbf{p}}+\sigma_{u}^{-2} \mathbf{A}^{21} \hat{\mathbf{u}}_{1}+\mathbf{W}_{2}{ }^{\prime} \mathbf{R}^{-1} \mathbf{W}_{2} \hat{\mathbf{u}}_{2} \\
+\sigma_{u}^{-2}\left(\mathbf{A}^{22}-\mathbf{A}_{22}^{-1}\right) \hat{\mathbf{u}}_{2}+\sigma_{u}^{-2} \mathbf{G}_{22}^{-1} \hat{\mathbf{u}}_{2}=\mathbf{W}_{2}{ }^{\prime} \mathbf{R}^{-1} \mathbf{y}
\end{gathered}
$$

which is identical to the $\mathbf{u}_{2}$ equation [49] of the singlestep GBLUP model.

Genotyped animals without phenotypes include both young and older animals that have no phenotypes. Young genotyped animals that do not have their own phenotypes are automatically evaluated by solving MME [47] of the single-step GBLUP model and MME [48] of the single-step SNP BLUP model. We wish to show that $\mathbf{u}_{2}$ equations for this group of animals are equal between the 2 single-step genomic models. By setting $\mathbf{y}=\mathbf{0}, \mathbf{p}=\mathbf{0}, \mathbf{b}=\mathbf{0}$, and $\mathbf{W}_{2}=\mathbf{0}$ for genotyped animals without phenotype, the $\mathbf{u}_{2}$ equation [49] of the single-step GBLUP is simplified to

$$
\sigma_{u}^{-2} \mathbf{A}^{21} \hat{\mathbf{u}}_{1}+\sigma_{u}^{-2}\left(\mathbf{A}^{22}+\mathbf{G}_{22}^{-1}-\mathbf{A}_{22}^{-1}\right) \hat{\mathbf{u}}_{2}=\mathbf{0} .
$$

As also shown by Liu et al. (2014), the $\mathbf{u}_{2}$ equation in MME [48] of the single-step SNP BLUP model for genotyped animals that have no own phenotypes $(\mathbf{y}=$ $0, \mathbf{p}=0, \mathbf{b}=0$, and $\mathbf{W}_{2}=0$ ) becomes

$\sigma_{u}^{-2} \mathbf{A}^{21} \hat{\mathbf{u}}_{1}+\sigma_{u}^{-2} \mathbf{A}^{22} \hat{\mathbf{u}}_{2}+\sigma_{u}^{-2}\left(\frac{1}{k}-1\right) \mathbf{A}_{22}^{-1} \hat{\mathbf{u}}_{2}-\sigma_{u}^{-2} \frac{1}{k} \mathbf{A}_{22}^{-1} \mathbf{Z} \hat{\mathbf{g}}=\mathbf{0}$.

Using $\mathbf{a}_{2}=\mathbf{u}_{2}-\mathbf{Z g}$ and equation [13] $\mathbf{A}_{22}^{-1} \hat{\mathbf{a}}_{2}=k \mathbf{G}_{22}^{-1} \hat{\mathbf{u}}_{2}$, equation [54] becomes

$$
\sigma_{u}^{-2} \mathbf{A}^{21} \hat{\mathbf{u}}_{1}+\sigma_{u}^{-2} \mathbf{A}^{22} \hat{\mathbf{u}}_{2}-\sigma_{u}^{-2} \mathbf{A}_{22}^{-1} \hat{\mathbf{u}}_{2}+\sigma_{u}^{-2} \mathbf{G}_{22}^{-1} \hat{\mathbf{u}}_{2}=\mathbf{0} .
$$

Equation [55] of the single-step SNP BLUP model is identical to equation [53] of the single-step GBLUP model. Thus, the 2 single-step genomic models with a residual polygenic effect are proven to be equivalent for the (young) genotyped animals without phenotypes.

Although the single- or multi-step GBLUP models do not directly estimate effects of SNP markers, SNP effect estimates can be obtained by converting GEBV of the genomic reference animals as given in equation [16], $\hat{\mathbf{g}}=(1-k) \mathbf{B Z} \mathbf{G}^{\prime} \mathbf{G}_{22}^{-1} \hat{\mathbf{u}}_{2}$. This equation is also applicable to the single-step GBLUP model. If some genotyped animals with phenotypes that are included in the $\mathbf{u}_{2}$ equation should be excluded from the genomic reference population (e.g., bull dams or elite cows with extreme phenotypic records), we need to modify $\mathbf{Z}, \mathbf{G}_{22}^{-1}, \hat{\mathbf{u}}_{2}$ accordingly.

Equation $\mathrm{g}$ in MME [48] of the single-step SNP BLUP model is

$$
\sigma_{u}^{-2}\left(\frac{1}{1-k} \mathbf{B}^{-1}+\frac{1}{k} \mathbf{Z}^{\prime} \mathbf{A}_{22}^{-1} \mathbf{Z}\right) \hat{\mathbf{g}}-\sigma_{u}^{-2} \frac{1}{k} \mathbf{Z}^{\prime} \mathbf{A}_{22}^{-1} \hat{\mathbf{u}}_{2}=\mathbf{0} .
$$

Applying $\mathbf{a}_{2}=\mathbf{u}_{2}-\mathbf{Z g}$ and rearranging [56],

$$
\frac{1}{1-k} \mathbf{B}^{-1} \hat{\mathbf{g}}=\frac{1}{k} \mathbf{Z}^{\prime} \mathbf{A}_{22}^{-1} \hat{\mathbf{a}}_{2},
$$

which results in the equation $\mathbf{g}$ as

$$
\hat{\mathbf{g}}=(1-k) \frac{1}{k} \mathbf{B Z}^{\prime} \mathbf{A}_{22}^{-1} \hat{\mathbf{a}}_{2} .
$$

Since $\mathbf{A}_{22}^{-1} \hat{\mathbf{a}}_{2}=k \mathbf{G}_{22}^{-1} \hat{\mathbf{u}}_{2}$ (equation [13]),

$$
\hat{\mathbf{g}}=(1-k) \mathbf{B} \mathbf{Z}^{\prime} \mathbf{G}_{22}^{-1} \hat{\mathbf{u}}_{2},
$$

which is identical to the SNP effect equation [16] of the GBLUP model.

Because the single-step GBLUP model does not provide the 2 components of GEBV, DGV, and RPG of the (young) genotyped animals without phenotypes, we can also apply equation [37], $\mathbf{Z}_{y} \hat{\mathbf{g}}=(1-k) \mathbf{G}_{y 2} \quad{ }_{D G V} \mathbf{G}_{22}^{-1} \hat{\mathbf{u}}_{2}$, to estimate DGV and equation [39], $\hat{\mathbf{a}}_{y}=k \mathbf{A}_{y 2} \mathbf{G}_{22}^{-1} \hat{\mathbf{u}}_{2}$, to predict RPG effect of the (young) genotyped animals without phenotypes, as the case for the multi-step GBLUP model. The decomposition of GEBV into 2 components is also applicable to the reference animals under the single-step GBLUP model.

In this paper we have proven that the 2 alternative genomic models, GBLUP and SNP BLUP, are equivalent when a residual polygenic effect is included in the genomic models. First, we verified the equivalence of the 2 multi-step genomic models for reference ani- 
mals, followed by (young) genotyped animals without phenotypes. Second, we extended our proof from the multi-step to single-step genomic models for genotyped animals with phenotypes as well as (young) genotyped animals without phenotypes. In addition, we proved the equivalent equations for SNP effect estimation by the 2 competing genomic models. For the GBLUP models, we provided formulas to convert GEBV to its 2 components, DGV and RPG effects. We have confirmed what has long been accepted as a fact that adding young genotyped animals into the multi-step GBLUP equations does not alter GEBV of the reference animals, suggesting the young genotyped animals can be evaluated in a posterior step to reduce the computing costs. We have also demonstrated for the multi-step genomic models that the genomic relationship among the young genotyped animals without phenotypes themselves has no influence on GEBV of the other young animals, indicating that the young genotyped animals can be evaluated group-wise or on a one-by-one basis. This justifies the frequent or continuous genomic evaluations (Alkhoder et al., 2014; Wiggans et al., 2015). In contrast to the multi-step genomic models, GEBV of young genotyped animals without phenotypes cannot be properly calculated in a posterior step under the single-step genomic models, because their GEBV are additionally dependent on their nongenotyped ancestors with phenotypes. An approximate procedure by Liu et al. (2014) may be used for calculating GEBV of the young genotyped animals without solving the complete set of equations.

We restricted our proofs to the BLUP genomic models and ignored Bayesian genomic models (Meuwissen et al., 2001; Wang et al., 2015). However, our assumption of the SNP effect distribution can be relaxed. For example, the matrix $\mathbf{B}$ may be a diagonal one, allowing some SNP markers having different variances. All the equations or formulas are still valid for a diagonal matrix $\mathbf{B}$.

In GBLUP evaluations, the observed genomic relationship matrix is usually combined with pedigree relationship matrix to obtain the final genomic relationship matrix. Different approaches have been developed in the literature to obtain an optimal genomic relationship matrix for the GBLUP model, such as centering and scaling the genomic relationship matrix (Strandén and Christensen, 2011; Forni et al., 2011; de Los Campos et al., 2013; Su et al., 2014). We did not address this issue in detail. However, if the modification of the genomic relationship matrix in the GBLUP models is done in a consistent way as setting up the regression coefficients on genotypes in the SNP BLUP models, the equivalence of the 2 groups of genomic models is retained.
In literature, many single-step GBLUP models have reduced the overestimation by changing the weights on inverse matrices of genomic and pedigree relationships, instead of weighting the original genomic and pedigree relationship matrices. For example, the computing algorithm for proven and young animals (APY, Misztal et al., 2014) weights the inverse matrices when applied to actual data (Masuda et al., 2015). These 2 different weighting procedures may lead to different genomic prediction. Further research is needed to understand these 2 weighting procedures.

We did not compare the computational (dis)advantages of the 2 competing genomic models, which are beyond the scope of this study. Although the single or multiple genomic GBLUP and SNP BLUP models are equivalent in theory, they differ markedly in computational efficiency and feasibility for very large genotyped populations. The SNP BLUP models are computationally more suitable for large data sets than the GBLUP models because they do not involve setting up or inverting large genomic relationship matrices. With ever increasing number of genotyped animals, inverting the genomic relationship matrix becomes less and less feasible. The new APY algorithm (Misztal et al., 2014; Masuda et al., 2015) can significantly ease this problem. When the number of genotyped animals exceeds the number of fitted SNP markers, GBLUP models may have to deal with the observed genomic relationship matrix that is not invertible. In contrast, the higher number of genotyped animals with phenotypes does not cause such a numerical problem to the SNP BLUP genomic models; instead it can increase reliabilities of the SNP effect estimates.

In addition, we did not address the issue of approximation of genomic reliability for both groups of genomic models (Strandén and Garrick, 2009), in which SNP allele frequencies played a role (Strandén and Christensen, 2011). Genomic reliabilities can be obtained by inverting the GBLUP equations for the genomic models with a RPG as for those without a RPG effect. The inclusion of a RPG into the SNP BLUP models has made the reliability calculation more complicated and difficult. This needs to be addressed in future.

We have proven in this study that the GBLUP and SNP BLUP models including a residual polygenic effect are equivalent for both multi- and single-step genomic predictions. The equivalence of the 2 alternative genomic models is valid for genotyped animals with phenotypes, including genomic reference animals, as well as for (young) genotyped animals without phenotypes. We have also demonstrated that the equations for SNP effect estimation are equivalent between the 2 genomic models. 


\section{ACKNOWLEDGMENTS}

The first author thanks Dairy Futures Cooperative Research Centre (Melbourne, Australia) for funding his sabbatical leave at AgriBio, La Trobe University (Melbourne, Australia).

\section{REFERENCES}

Alkhoder, H., Z. Liu, Th. Bach, E. Pasman, and F. Reinhardt. 2014. A continuous genomic evaluation system for German Holsteins. Interbull Bull. 48:100-104.

Christensen, O. F., and M. S. Lund. 2010. Genomic prediction when some animals are not genotyped. Genet. Sel. Evol. 42:2.

de Los Campos, G., J. M. Kickey, R. Pong-Wong, H. Daetwyler, and M. P. L. Calus. 2013. Whole-genome regression and prediction methods applied to plant and animal breeding. Genetics 193:327345 .

Fernando, R., J. C. M. Dekkers, and D. J. Garrick. 2014. A class of Bayesian methods to combine large numbers of genotyped and non-genotyped animals for whole-genome analyses. Genet. Sel. Evol. 46:50.

Forni, S., I. Aguilar, and I. Misztal. 2011. Different genomic relationship matrices for single-step analysis using phenotypic, pedigree and genomic information. Genet. Sel. Evol. 43:1.

Harville, D. A. 1997. Matrix Algebra from a Statistician's Perspective. Springer-Verlag, New York, NY.

Hayes, B. J., P. J. Bowman, A. J. Chamberlain, and M. E. Goddard. 2009. Invited review: Genomic selection in dairy cattle: Progress and challenges. J. Dairy Sci. 92:433-443.

Jairath, L., J. C. M. Dekkers, L. R. Schaeffer, Z. Liu, E. B. Burnside, and B. Kolstad. 1998. Genetic evaluation for herd life in Canada. J. Dairy Sci. 81:550-562.

Legarra, A., and V. Ducrocq. 2012. Computational strategies for national integration of phenotypic, genomic, and pedigree data in a single-step best linear unbiased prediction. J. Dairy Sci. 95:4629 4645 .

Liu, Z., M. E. Goddard, F. Reinhardt, and R. Reents. 2014. A singlestep genomic model with direct estimation of marker effects. J. Dairy Sci. 97:5833-5850.
Liu, Z., F. R. Seefried, R. Reinhardt, S. Rensing, G. Thaller, and R. Reents. 2011. Impacts of both reference population size and inclusion of a residual polygenic effect on the accuracy of genomic prediction. Genet. Sel. Evol. 43:19.

Masuda, Y., I. Misztal, S. Tsuruta, D. A. L. Lourenco, B. O. Fragomeni, A. Legarra, I. Aguilar, and T. J. Lawlor. 2015. Single-step genomic evaluations with $570 \mathrm{~K}$ genotyped animals in US Holsteins. Interbull Bull. 49:85-89.

Meuwissen, T. H. E., B. J. Hayes, and M. E. Goddard. 2001. Prediction of total genetic value using genome-wide dense marker maps. Genetics 157:1819-1829.

Misztal, I., A. Legarra, and I. Aguilar. 2009. Computing procedures for genetic evaluation including phenotypic, full pedigree, and genomic information. J. Dairy Sci. 92:4648-4655.

Misztal, I., A. Legarra, and I. Aguilar. 2014. Using recursion to compute the inverse of the genomic relationship matrix. J. Dairy Sci. 97:3943-3952.

Strandén, I., and O. F. Christensen. 2011. Allele coding in genomic evaluation. Genet. Sel. Evol. 43:25.

Strandén, I., and D. J. Garrick. 2009. Technical note: Derivation of equivalent computing algorithms for genomic predictions and reliabilities of animal merit. J. Dairy Sci. 92:2971-2975.

Su, G., O. F. Christensen, L. Janss, and M. S. Lund. 2014. Comparison of genomic predictions using genomic relationship matrices built with different weighting factors to account for locus-specific variances. J. Dairy Sci. 97:6547-6559.

Su, G., P. Madsen, U. S. Nielsen, E. A. Mäntysaari, G. P. Aamand, O. F. Christensen, and M. S. Lund. 2012. Genomic prediction for Nordic Red Cattle using one-step and selection index blending. J. Dairy Sci. 95:909-917.

Taylor, J. F. 2014. Implementation and accuracy of genomic selection. Aquaculture 420-421:S8-S14.

VanRaden, P. M. 2008. Efficient methods to compute genomic predictions. J. Dairy Sci. 91:4414-4423.

Wang, T., Y. P. Chen, M. E. Goddard, T. H. E. Meuwissen, K. E. Kamper, and B. Hayes. 2015. A computationally efficient algorithm for genomic prediction using a Bayesian model. Genet. Sel. Evol. 47:34.

Wiggans, G., P. M. VanRaden, and T. A. Cooper. 2015. Technical note: Rapid calculation of genomic evaluations for new animals. J. Dairy Sci. 98:2039-2042. 